\title{
Optimal Decentralized Microgrid Coordination via the Schur's Complement and S-Procedure
}

\author{
Mithun Mallick, Student member, IEEE, Pirathayini Srikantha, Member, IEEE
}

\begin{abstract}
The evolving landscape of the electricity sector along with increasing environmental concerns necessitate modern power grids to be more efficient, sustainable and adaptive. Microgrids are typically composed of distributed energy sources which have great potential for enabling energy independence, sustainability and flexibility. However, practical difficulties that deter the widespread deployment of microgrids include the unpredictability of local generation sources (e.g. renewables) and the lack of inertia that is naturally present in systems containing bulk synchronous plants. In this paper, we propose a near real-time microgrid coordination algorithm that allows actuating components to adapt to changing system conditions. We account for the electrical dependencies and limits in microgrid systems by constructing voltage/current balance relations in the $d q 0$ frame and applying strategic decompositions to invoke the Schur's complement and S-procedure that allow for zero duality gap. We highlight the convergence, feasibility and scalability features of the proposed decentralized algorithm via theoretical and comparative practical simulation studies.
\end{abstract} tion

Index Terms-Microgrids, Decomposition, Convex Optimiza-

\section{INTRODUCTION}

Microgrids typically consist of distributed energy resources (DERs) such as photovoltaics and wind turbines that generate electricity by tapping onto locally available natural resources which is then delivered at low losses to power consumers residing at close physical proximity. These systems operate in either grid-connected or stand-alone modes. As the microgrid operating in stand-alone mode lacks the inertia introduced by the main grid, highly fluctuating power demand/suppy will lead to inefficiencies and instabilities that can be overcome by applying appropriate optimal steady-state and transient control mechanisms [1]-[5]. In this paper, we focus specifically on steady-state coordination over near real-time horizons (e.g. seconds) to closely account for rapidly changing conditions in the microgrid. This remains an open research challenge mainly due to the non-convexities present in power flow relations which must be accurately incorporated for feasible microgrid operations [6], [7]. Thus, our main contribution in this paper is the proposal of a decentralized DER coordination method that accounts for physical power balance relations with convergence guarantees.

Existing work in this area can be loosely classified into centralized and decentralized strategies. In centralized coordination approaches, a single entity computes optimal steady-state

M. Mallick and P. Srikantha are with the Department of Electrical and Computer Engineering, Western University, London, ON, Canada email: mmallic2@uwo.ca and psrikan@uwo.ca. set-points for actuating electrical nodes. For this, the coordinating entity must be equipped with knowledge of the entire microgrid configuration along with local power demand/supply trends. Highly-granular coordination becomes intractable due to the sheer scale of parameters that must be constantly accrued by the central entity and the computational complexity resulting from non-convex power flow relations. To overcome these issues, forecast models of renewable generation and consumer demands are utilized to perform computations over longer time horizons. These models are typically associated with significant error margins and can lead to inefficiencies [8]. Non-convexities are addressed via relaxation or heuristic techniques [9]-[11]. Under certain assumptions and conditions, these relaxations can be proven to be exact as show in references [12], [13]. These assumptions and conditions can be limiting. Additionally, the centralized model is associated with limited flexibility as the coordinating entity must be updated with every system change. As such, reference [11] proposes a master-slave coordination strategy in which a dominant DER performs voltage regulation while other units perform loadsharing. This proposal, inspired by the conventional power system, is contingent upon the presence of this central DG and its relative dominance with respect to other system entities.

Decentralized coordination strategies empower individual components in the microgrid (i.e. DERs) with actuation capabilities based on local state measurements and/or iterative information exchanges with peers. This paradigm eliminates issues in the centralized model such as single point of failure, concentrated computational overheads and limited flexibility. Droop-based coordination methods (e.g. [6]) involve no communication and entail the sampling of local state measurements which are utilized to compute actuation that suppresses transience. As the global system state is not considered in the actuation decisions, inefficiencies can exist. Other strategies leverage communication processes to exchange information amongst peer nodes in the microgrid where data signals are designed via consensus and incremental-cost based droop techniques [14]-[19]. These proposals also apply relaxations to eliminate non-convex constraints for guaranteeing convergence. For instance, reference [19] applies a semi-definite (SD) relaxation and eliminates a non-convex rank one matrix constraint. Then, alternating direction method of multipliers (ADMM) is applied to solve the problem in a decentralized manner. The resulting solution is not feasible if it does not satisfy the eliminated rank one matrix constraint.

In this paper, the proposed microgrid coordination algorithm is a novel departure from existing literature as we are able to employ strategic decomposition and problem reformulations 
to account for non-convexities that cause intractability and solve the coordination problem in a decentralized manner. Specifically, the three main contributions in this paper are the following: 1) We are able to decompose the original nonconvex coordination problem into sub-problems for each actuating entity by strategically utilizing a variable transformation and the operator-splitting method ADMM so that an exact relaxation can be applied to the sub-problems via the Schur's Complement and S-Procedure; 2) Due to the convex nature of this transformation, the proposed decentralized algorithm that involves iterative information exchanges with only directly connected actuating DER nodes is guaranteed to converge under non-restrictive conditions; and 3) Convergence speed of the algorithm is proportional to the number of actuating nodes in the system which allows for near real-time coordination as demonstrated by simulation studies on realistic islanded threephase microgrid systems. Comparative studies with recent work are also included to highlight features of the proposed algorithm.

The remainder of this paper is organized as follows. Section II introduces the system model utilized in this paper to represent the communication and electrical attributes of a cyber-physical microgrid. In Section III, proposed decentralized microgrid coordination algorithm is introduced along with theoretical studies. Section IV validates the proposal via simulation and comparative studies conducted on realistic microgrids. Finally, the paper is concluded in Section V.

\section{SySTEM MODEL}

In today's era of the internet of things (IoT), power entities are typically cyber-enabled and are equipped with intelligence to compute local actuation by way of information exchanges [20]. As such in this section, we present the model that we utilize to represent the communication processes and physical attributes of a cyber-physical microgrid system. Then, we formulate the optimal microgrid coordination problem.

\section{A. A Cyber-physical Microgrid}

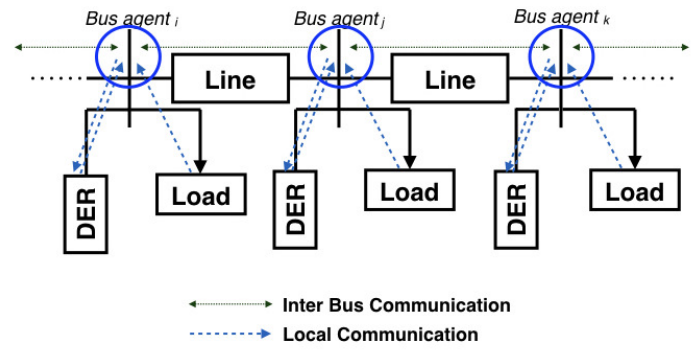

Fig. 1: A cyber-physical microgrid.

A microgrid is composed of active and passive elements (e.g. generation units and power consumers) residing at close geographical proximity as illustrated in Fig. 1. Each bus in the microgrid represents a sub-system consisting of a load, an electrical line and a DER. The DER is composed of an energy source that connects to a storage system (to buffer the power supplied by the source) which then interfaces with an inverter. These sub-systems are then connected to one another via power lines. We adopt a cyber-physical model where each subsystem in the microgrid is represented by a bus agent that is composed of an intelligent module with communication and computational capabilities. These bus agents will interact with neighbouring buses every 100 milliseconds (ms) and exchange information as necessary. This information exchange frequency accounts for typical delays incurred in the wireless communication medium (between $8 \mathrm{~ms}$ to $30 \mathrm{~ms}$ for single hop data exchange) [21]. The bus agents refine local solutions based on the information received from peer nodes and local state measurements (current, voltage, etc.). Once these computations converge, each bus agent will communicate the voltage set-point to the local DER. This process is repeated every time an impending change in load or the DER generation capacity is expected. During these instances, the relevant entity (DER or load) alerts the appropriate bus agent of the change.

\section{B. Physical Microgrid Attributes}

The physical microgrid model and the associated system parameters utilized in this paper are adopted from reference [1]. In this model, each sub-system is considered to be a building block of the microgrid that connect to one another to form the entire microgrid network. Fig. 2 presents a singleline diagram of sub-system $i \in n$ where $n$ represents the total number of sub-systems in the microgrid. Each sub-system is composed of three main components: 1) DER; 2) load; and 3) line.

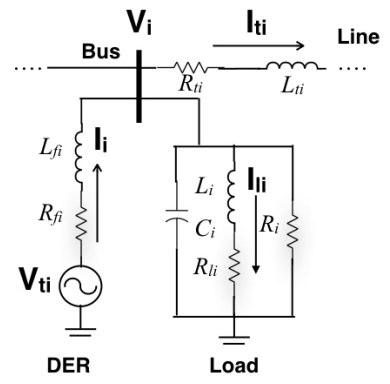

Fig. 2: Single-line diagram of sub-system $i$.

The DER is represented by a voltage source converter (VSC) for which the voltage and power ratings are set to be $0.6 \mathrm{kV}$ and 1.6 MVA respectively. According to the survey presented in reference [22], these ratings are utilized in practical microgrid systems such as those deployed by BC Hydro and Hydro Quebec that are compliant with the IEEE standard listed in reference [23]. The voltage at the VSC is denoted by the variable $V_{t i}$ and this set-point is actuated by the bus agent. The VSC is connected to an RL filter with a quality factor of 50. This filter is utilized to smooth the current $I_{i}$ flowing from the VSC into the microgrid [1], [24]. The VSC and filter compose the DER unit which connects to the microgrid bus via a $0.6 / 13.8 \mathrm{kV}$ delta-wye step-up transformer [1]. The next component in the sub-system is the load which is modelled as three parallel branches consisting of lumped inductive, capacitive and resistive elements [25]. In this configuration, the branch consisting of the resistor $R_{i}$ represents predominantly resistive loads such as heaters, 
filaments, etc. The RL branch composed of resistor $R_{l i}$ and inductor $L_{i}$ accounts for motor loads which constitute a wide proportion of loads in distribution feeders [24]. The final branch consisting of the capacitor $C_{i}$ captures stray capacitances, capacitive power factor correction devices and/or highly capacitive loads such as electrostatic precipitators. We intend to extend this model to include constant power loads in future work. The variable $I_{l i}$ denotes the current flowing across the RL branch. The next component is the tie-line connecting this sub-system to other sub-systems. The line inductance and capacitance parameters are represented by $R_{t i}$ and $L_{t i}$. Values taken by these parameters are associated with low reactance to resistance ratio as typical in standard distribution systems [26]. The current flowing through the line is denoted as $I_{t i}$. Finally, the voltage $V_{i}$ at the bus connecting the DER, load and line must remain within \pm 0.05 p.u. of the nominal voltage.

Hence, each sub-system $i$ is associated with four state variables $\left(V_{i, a b c}, I_{i, a b c}, I_{l i, a b c}, I_{t i, a b c}\right)$ where $a b c$ denotes the three phases in the alternating current (AC) representation of the microgrid system. The direct-quadrature-zero $d q 0$ frame of reference is widely utilized in microgrid control (e.g. [1]) instead of the $a b c$ frame as the state-space equations of the circuit can be represented as a first-order linear system in terms of voltage and current variables. In typical voltage phasor models, there are non-convexities associated with power balance relations. The conversion of a state variable $K$ from the $a b c$ to the $d q 0$ frame is obtained via the Park's transformation that is applied around a rotating frame of reference with frequency $\omega[27]$ :

$$
K_{d q}=\frac{2}{3}\left(\begin{array}{ccc}
\cos \theta & \cos \left(\theta-\frac{2}{3} \pi\right) & \cos \left(\theta+\frac{2}{3} \pi\right) \\
-\sin \theta & -\sin \left(\theta-\frac{2}{3} \pi\right) & -\sin \left(\theta+\frac{2}{3} \pi\right) \\
\frac{1}{\sqrt{2}} & \frac{1}{\sqrt{2}} & \frac{1}{\sqrt{2}}
\end{array}\right) K_{a b c}
$$

$\theta$ is the phase angle associated with the rotating frame and this must be tracked by every bus agent operating in the $d q 0$ frame. This phase angle is synchronized amongst bus agents via internal crystal oscillators that are maintained within each bus agent. Each oscillator will generate phase angle at time $t$ according to $\theta(t)=\int_{0}^{t} \omega_{0} d \tau$ where $f_{0}=\frac{2 \pi}{\omega_{0}}$ is the nominal frequency of the microgrd. This phase angle is then utilized to perform the Park's transformation. Synchronization between local oscillators in bus agents are maintained via a global synchronizing signal that each DER receives every one second from Global Positioning Systems (GPS) to prevent drifts over extended periods [28], [29]. The crystal oscillators are economical and highly accuracies as well [30]. This openloop frequency control method has been utilized in existing literature such as reference [1]. With this variable transformation, voltage and current variables in the $d q 0$ frame are twodimensional (i.e. $\mathbb{R}^{2 \times 1}$ ), real and linearly related in steadystate conditions. To show this, the state-space equations in the $d q 0$ frame representing the electrical interdependencies in each sub-system $i$ are first derived by applying the Kirchhoff's voltage and current laws to the single-line circuit presented in Fig. 2:

$$
\begin{aligned}
& \frac{d V_{i, d q}}{d t}=-\frac{V_{i, d q}}{R_{i} C_{i}}+\left[\begin{array}{cc}
0 & w \\
-w & 0
\end{array}\right] V_{i, d q}+\frac{I_{i, d q}}{C_{i}}-\frac{I_{L i, d q}}{C_{i}}-\frac{I_{t i, d q}}{C_{i}}+\frac{I_{t i-1, d q}}{C_{i}} \\
& \frac{d I_{i, d q}}{d t}=-\frac{V_{i, d q}}{L_{f i}}-\frac{R_{f i}}{L_{f i}} I_{i, d q}+\left[\begin{array}{cc}
0 & w \\
-w & 0
\end{array}\right] I_{i, d q}+\frac{V_{t i, d q}}{L_{f i}} \\
& \frac{d I_{L i, d q}}{d t}=\frac{V_{i, d q}}{L i}-\frac{R_{l i}}{L_{i}} I_{L i, d q}+\left[\begin{array}{cc}
0 & w \\
-w & 0
\end{array}\right] I_{L i, d q} \\
& \frac{d I_{t i, d q}}{d t}=\frac{V_{i, d q}}{L_{t i}}-\frac{V_{i+1, d q}}{L_{t i}}-\frac{R_{t i}}{L_{t i}} I_{t i, d q}+\left[\begin{array}{cc}
0 & w \\
-w & 0
\end{array}\right] I_{t i, d q}
\end{aligned}
$$

Neighbours of sub-system $i$ on the left and right are indexed as $i-1$ and $i+1$ respectively. We consider serially connected microgrid as in reference [1] for notational convenience without loss of generality. At steady-state, the differential terms will be 0 which will result in a set of linear voltage and current balance relations.

\section{Formulation of Optimal Coordination Problem}

In the optimal microgrid coordination problem considered in this paper, we aim to minimize the cost of power supplied by DERs in the system. Typically, there is no fuel cost associated with renewable DERs (e.g. solar and wind generation). The DERs considered in this paper are interfaced with storage systems and therefore are dispatchable. As outlined by reference [31], there are costs associated with the installation, investment and reliability of these energy sources. These costs can be recovered via the price assigned for power generated by these DERs. Furthermore, with the integration of technologies such as BlockChain, peer-to-peer energy trading is now possible [32]. Price can be assigned to power generated by these entities to facilitate this decentralized energy trading process. Moreover, this will further incentivize increased deployment of renewables by power consumers. We will investigate extending the proposed algorithm for any cost function in future work.

As we consider voltage and current variables in the $d q 0$ frame in our system model, power $P_{i}$ supplied by DER $i$ should be expressed in terms of these variables. This is done by first observing that [33]:

$$
P_{i}=\frac{3}{2} V_{t i, d q}^{\prime} I_{i, d q}
$$

$I_{i, d q}$ can be expressed in terms of $V_{t i}$ and $V_{i}$ by re-arranging the relation in line 2 of Eq. 1 in steady-state (i.e. $\frac{d I_{i, d q}}{d t}=0$ ):

$$
I_{i, d q}=\left[\begin{array}{cc}
R_{f i} & -w L_{f i} \\
w L_{f i} & R_{f i}
\end{array}\right]^{-1}\left[V_{t i, d q}-V_{i, d q}\right]
$$

Substituting Eq. 3 into Eq. 2 to obtain an expression for $P_{i}$ in terms of $V_{t i, d q}$ and $V_{i, d q}$ and assuming a linear cost function $K_{i} P_{i}$ where $K_{i} \geq 0$ is a constant parameter, we derive the cost function $f_{i}\left(V_{i, d q}, V_{t i, d q}\right)$ for generation in sub-system $i$.

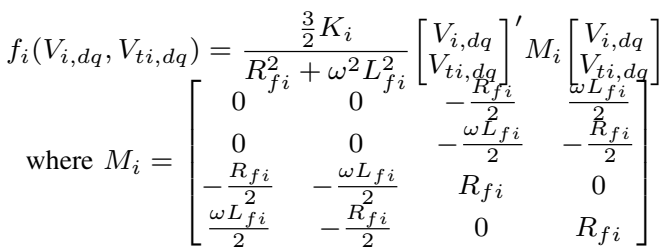

The constant term $\frac{1}{R_{f i}^{2}+\omega^{2} L_{f i}^{2}}$ stems from the determinant of the inverse matrix in Eq. 3 which is positive (quadratic real 
terms). It is important to note that the Hessian of the matrix $M_{i}$ is not necessarily positive semi-definite due to the low $X / R$ ratio typical in microgrids. Thus, $f_{i}\left(V_{i, d q}, V_{t i, d q}\right)$ can be non-convex. We formulate the optimal microgrid coordination problem composed of this objective in $\mathcal{P}_{C}$ :

$$
\begin{aligned}
& P_{C}: \underset{V, I, I_{L}, I_{t}, V_{t}}{\operatorname{minimize}} \sum_{i \in n} f_{i}\left(V_{i, d q}, V_{t i, d q}\right) \\
& \text { subject to: } \forall i \in n \\
& \frac{-V_{i, d q}}{R_{i} C_{i}}+\left[\begin{array}{cc}
0 w \\
-w & 0
\end{array}\right] V_{i, d q}+\frac{I_{i, d q}-I_{L i, d q}-I_{t i, d q}+I_{t i-1, d q}}{C_{i}}=0 \\
& -\frac{V_{i, d q}}{L_{f, i}}-\frac{R_{f, i}}{L_{f, i}} I_{i, d q}+\left[\begin{array}{cr}
0 & w \\
-w & 0
\end{array}\right] I_{i, d q}+\frac{V_{t i, d q}}{L_{f i}}=0 \\
& \frac{V_{i, d q}}{L i}-\frac{R_{l i}}{L_{i}} I_{L i, d q}+\left[\begin{array}{cc}
0 w \\
-w & 0
\end{array}\right] I_{L i, d q}=0 \\
& \frac{V_{i, d q}}{L_{t i}}-\frac{V_{i+1, d q}}{L_{t i}}-\frac{R_{t i}}{L_{t i}} I_{t i, d q}+\left[\begin{array}{cc}
0 & w \\
-w & 0
\end{array}\right] I_{t i, d q}=0 \\
& I_{i, d q} \mathbb{I}_{e m p t y}(i)=0 \\
& 0.95^{2} \leq V_{i, d q}^{\prime} V_{i, d q} \leq 1.05^{2}
\end{aligned}
$$

Constraints $(C 1)-(C 4)$ represent voltage and current balance equations in steady-state. As these are linear relations, these are convex constraints. The demand at each bus is modelled in a passive manner via the load parameters $R_{i}, R_{L i}, C_{i}, L_{i}$ which affects the current flowing into the three load branches. These parameters will be updated every-time $\mathcal{P}_{C}$ is solved to reflect the ongoing changes in demand in the microgrid system. $\mathbb{I}_{\text {empty }}(i)$ is an indicator variable that is set to 1 if storage in DER $i$ is empty and 0 otherwise. Thus, $(C 5)$ captures variability in generation capacities of DERs. (C6) represents the upper and lower limits on bus voltage magnitude which are squared in order to eliminate the square root associated with the voltage magnitude term. The upper limit of this constraint is convex as the Hessian of the inequality in the standard form (i.e. $f(x) \leq 0$ ) is positive semi-definite. On the other hand, the Hessian associated with the lower limit of this constraint is not positive semi-definite. This constraint is not convex and it can be shown that the formal definition of a convex set $\mathcal{S}$ (i.e. $\theta x+(1-\theta) y \in \mathcal{S}$ where $x \in \mathcal{S}, y \in \mathcal{S}, \theta \in[0,1]$ [35]) does not hold.

Thus, $\mathcal{P}_{C}$ consists of two instances of non-convexities: 1) Cost of power generated by DERs; and 2) Lower voltage magnitude limit. These non-convexities render directly solving $\mathcal{P}_{C}$ NP-hard which translates to intractability for near realtime computations in large microgrid systems (i.e. $n$ is large) [35]. We tackle the issue of non-convexity by employing strategic decomposition that allows for the application of exact convex relaxations as discussed next.

\section{Proposed Decentralized Algorithm}

In this paper, we are proposing a highly granular and flexible microgrid coordination algorithm that can accommodate the fluctuating nature of generation/demand and the seamless plug-and-play integration of diverse power components. For this, it is necessary to solve $\mathcal{P}_{C}$ frequently (e.g. timescale of seconds) to adapt to ongoing system changes and in a decentralized manner to eliminate concentrated overheads. As $\mathcal{P}_{C}$ is a non-convex problem, decentralized techniques will be prone to issues such as divergence and sub-optimality. On the other hand, convex relaxations can result in approximations that may violate important system limits (e.g. bus voltage, etc.). To overcome these issues, we utilize ADMM to strategically decompose $\mathcal{P}_{C}$ into a set of sub-problems solved by each bus agent so that exact convex relaxations can be applied to these for guaranteeing feasibility, tractability and convergence.

The application of ADMM to decompose optimal power flow problems is not a novel concept as illustrated in recent works such as [17], [19]. Although we adopt main elements of ADMM proposed in the context of power systems (e.g. local/perspective variables), we differ in two main respects: 1) Use of a linear $d q 0$-frame of reference in the problem formulation; and 2) Strategic decomposition of the problem for allowing an exact convex relaxation. After decomposing $\mathcal{P}_{C}$, we apply a convex relaxation via the Schur's complement to the dual of each sub-problem and then utilize the S-procedure to prove the exact nature of this relaxation. The iterative refinement of local VSC setpoints obtained by solving these convex sub-problems will result in linear convergence of the system. Details on our proposed decentralized algorithm are presented next.

\section{A. Decoupled Optimization Problem}

The decomposition of problem $\mathcal{P}_{C}$ for each bus agent $i$ is not straight-forward as constraints such as $(C 1)$ and $(C 4)$ are not separable. These contain variables such as $I_{t i-1, d q}$ and $V_{i+1, d q}$ that belong to neighbouring sub-systems (i.e. bus agent $i-1$ and $i+1)$. In order to overcome this issue, we adopt the notion of perspective variables introduced in reference [17]. Each bus agent $i$ will maintain two sets of variables: 1) Local variables $x_{i}$; and 2) Perspective variables $y_{i}$. Local variables consist of all state variables that belong to sub-system $i$ :

$$
x_{i}=\left\{V_{i}^{x}, I_{i}^{x}, I_{L i}^{x}, I_{t i}^{x}, V_{t i}^{x}\right\}
$$

The sub-script $d q$ is omitted for brevity. Henceforth, all variables are referred to in the $d q 0$ frame of reference unless otherwise specified. (C6) imposes limits on individual variables belonging to sub-system $i$. The lower limit introduces the non-convexity in the bus voltage magnitude constraint. We define, all feasible points defined by this lower limit to form the set $\mathcal{X}_{i}$. Hence, local variables $x_{i}$ must belong to the set $\mathcal{X}_{i}$ (i.e. $x_{i} \in \mathcal{X}_{i}$ ). Perspective variables maintained by bus agent $i$ are guesses of actual variables. The remaining constraints are $(C 1)-(C 5)$ and the upper limit associated with $(C 6)$. These contain variables belonging to neighbouring agents. Hence, local perspectives of variables in these equations are maintained in the set $y_{i}$ :

$$
y_{i}=\left\{V_{i, i}^{y}, V_{i+1, i}^{y}, I_{i, i}^{y}, I_{L i, i}^{y}, I_{t i, i}^{y}, I_{t i-1, i}^{y}, V_{t i, i}^{y}\right\}
$$

The subscripts of these $y$ variables contain two indices where the first index reflects the perspective of and the second index represents the perspective from. For instance, $I_{t i-1, i}^{y}$ is the line current of sub-system $i-1$ from the perspective of bus agent $i$. These variables must satisfy the voltage and current balance relations in $(C 1)-(C 5)$ along with the upper limit on bus voltage magnitude. All feasible points that heed these relations form the set $\mathcal{Y}_{i}$. Thus, the perspective variables $y_{i}$ 
must belong to the set $\mathcal{Y}_{i}$ (i.e. $y_{i} \in \mathcal{Y}_{i}$ ). Now, the problem $\mathcal{P}_{C}$ is separable in terms of the local and perspective variables for each bus agent. However, for these to represent the original problem in $\mathcal{P}_{C}$, the actual variables must be equal to the guesses/perspectives maintained by the bus agents. Thus, in each sub-system $i$, a consensus must be established between $y_{i}$ and $x_{i}, x_{i+1}$ and $x_{i-1}$. Problem $\mathcal{P}_{S}$ is formulated in terms of these local and perspective variables along with consensus constraints:

$$
\begin{aligned}
& \mathcal{P}_{S}: \underset{x \in \mathcal{X}, y \in \mathcal{Y}}{\operatorname{minimize}} \sum_{i \in n} f_{i}\left(x_{i}\right) \\
& \text { s.t. } \forall i \in n \\
& V_{i, i}^{y}=V_{i}^{x}, V_{i+1, i}^{y}=V_{i+1}^{x}, I_{i, i}^{y}=I_{i}^{x}, I_{L i, i}^{y}=I_{L i}^{x}, \\
& I_{t i, i}^{y}=I_{t i}^{x}, I_{t i-1, i}^{y}=I_{t, i-1}^{x}, V_{t i, i}^{y}=V_{t i}^{x}
\end{aligned}
$$

Now, with the addition of the consensus constraints, problem $\mathcal{P}_{S}$ will be equivalent to $\mathcal{P}_{C}$. In order to decompose $\mathcal{P}_{S}$ into sub-problems solved by each bus agent, we construct the augmented Lagrangian $\mathcal{L}_{\rho}^{S}\left(x_{i}, y_{i}, v_{i}\right)$ for this problem where $M x-N y$ represents the consensus constraints in $\mathcal{P}_{S}, \nu$ is the dual variable associated with the consensus constraints and $\rho>0$ is the penalty parameter that imposes strict convexity of the Lagrangian.

$$
\begin{aligned}
& \mathcal{L}_{\rho}^{S}(x, y, \nu)=\sum_{i \in n} f_{i}\left(x_{i}\right)+v^{T}(M x-N y)+\frac{\rho}{2}\|M x-N y\|_{2}^{2} \\
& =\sum_{i \in n} f_{i}\left(V_{t i}^{x}, V_{i}^{x}\right)+\nu_{i, i}^{V}\left(V_{i}^{x}-V_{i, i}^{y}\right)+\nu_{i+1, i}^{V}\left(V_{i+1}^{x}-V_{i+1, i}^{y}\right) \\
& +\nu_{i, i}^{I}\left(I_{i}^{x}-I_{i, i}^{y}\right)+\nu_{i, i}^{I_{L}}\left(I_{L i}^{x}-I_{L i, i}^{y}\right)+\nu_{i, i}^{I_{t}}\left(I_{t i}^{x}-I_{t i, i}^{y}\right) \\
& +\nu_{i-1, i}^{I_{t}}\left(I_{t, i-1}^{x}-I_{t i-1, i}^{y}\right)+\nu_{i, i}^{V_{t}}\left(V_{t i}^{x}-V_{t i, i}^{y}\right)+\frac{\rho}{2}\left(V_{i}^{x}-V_{i, i}^{y}\right)^{2} \\
& +\frac{\rho}{2}\left(V_{i+1}^{x}-V_{i+1, i}^{y}\right)^{2}+\frac{\rho}{2}\left(I_{i}^{x}-I_{i, i}^{y}\right)^{2}+\frac{\rho}{2} I_{L}\left(I_{L i}^{x}-I_{L i, i}^{y}\right)^{2} \\
& +\frac{\rho}{2}\left(I_{t i}^{x}-I_{t i, i}^{y}\right)^{2}+\frac{\rho}{2}\left(I_{t, i-1}^{x}-I_{t i-1, i}^{y}\right)^{2}+\frac{\rho}{2}\left(V_{t i}^{x}-V_{t i, i}^{y}\right)^{2}
\end{aligned}
$$

Each bus agent will maintain two versions of the Lagrangian function locally which are optimized over $x_{i}$ and $y_{i}$ respectively. Terms not relevant to the optimization being conducted are discarded as these become constant parameters that do not affect the optimization process. Thus, at every iteration $k$, each bus agent $i$ sequentially solves the Lagrangian as follows:

$$
\begin{aligned}
x_{i}^{k+1} & =\underset{x_{i} \in \mathcal{X}_{i}}{\operatorname{argmin}} \mathcal{L}_{p}^{S}\left(x, y^{k}, \nu^{k}\right) \\
y_{i}^{k+1} & =\underset{y_{i} \in \mathcal{Y}_{i}}{\operatorname{argmin}} \mathcal{L}_{p}^{S}\left(x^{k+1}, y, \nu^{k}\right) \\
v_{i}^{k+1} & =\nu_{i}^{k}+\rho\left(M x^{k+1}-N y^{k+1}\right)
\end{aligned}
$$

where variables marked with the superscript $k$ represent values computed in the preceding iteration which are held constant in the current iteration. Prior to update $(U 1)$, bus agent $i$ exchanges $\nu^{k}$ computed in iteration $k$ with neighbouring bus agents. Similarly for updates $(U 2)$ and $(U 3)$, bus agent $i$ exchanges $x_{k+1}$ and $y_{k+1}$ computed in $(U 1)$ and $(U 2)$ respectively with peers. Thus, it is clear that every bus agent iteratively refines local and perspective variables via three sets of information exchanges with directly connected neighbouring bus agents and this is a decentralized process requiring communication with nodes located at close proximity. This process is repeated until the residual of the consensus constraint $\|M x-N y\|_{2}^{2}$ falls below a pre-defined threshold $\epsilon$. At convergence, the consensus constraint $M x-N y=0$ which will result in the dual term $v^{T}(M x-N y)$ and the augmented term $\frac{\rho}{2}\|M x-N y\|_{2}^{2}$ in $\mathcal{L}_{\rho}^{S}(x, y, \nu)$ to be 0 which will result in the Lagrangian reducing to the original objective in $\mathcal{P}_{S}$ [38]. However, since the set $\mathcal{X}_{i}$ and the Lagrangian function $\mathcal{L}_{\rho}^{S}\left(x_{i}, y_{i}^{k}, \nu_{i}^{k}\right)$ which consists of $f_{i}\left(V_{i, d q}, V_{t i, d q}\right)$ are both not convex, this iterative decentralized update process is not guaranteed to converge to the globally optimal solution [18]. The problem solved in $(U 2)$ is convex as $\mathcal{Y}_{i}$ is composed of linear voltage/current balance constraints and convex upper bounds on bus voltage magnitude limits with the objective $\mathcal{L}_{p}^{S}\left(x^{k+1}, y, \nu^{k}\right)$ composed of quadratic variables with positive coefficients.

\section{B. Eliminating Non-convexities}

Thus, we focus next on applying transformations that can convert $(U 1)$ into an exact convex relaxation. First, we observe that the general form of the optimization problem in $(U 1)$ is:

$$
\begin{aligned}
& \mathcal{P}_{U 1}^{i}: \underset{x_{i} \in \mathcal{X}_{i}}{\operatorname{minimize}} \frac{1}{2} x_{i}^{T}\left(A_{0}^{i}+B_{0}^{i}\right) x_{i}+C_{0}^{i} x_{i} \\
& \text { s.t. } \underline{D_{1}^{i}} \leq \frac{1}{2} x_{i}^{T} A_{1}^{i} x_{i}
\end{aligned}
$$

where $A_{0}^{i}$ is the coefficient $\frac{\frac{3}{2} K_{i}}{R_{f i}^{2}+\omega^{2} L_{f i}^{2}} M_{i}$ from the cost function $f_{i}\left(V_{t i}, V_{i}\right) . B_{0}^{i}$ is a diagonal matrix containing the coefficients of quadratic terms in $\mathcal{L}_{\rho}^{S}$ pertaining to $x_{i}$. The general structure of $B_{0}^{i}$ is $2 \operatorname{diag}(2 \rho, 2 \rho, \rho, \rho, \rho, \rho, 2 \rho, 2 \rho, \rho, \rho)$ for all subsystems except for subsystems located at the ends of the physical microgrid network. For a subsystem having no neighbour $i+1$, the $7^{t h}$ and $8^{t h}$ elements in $B_{0}^{i}$ are set to $2 \rho$. Similarly for a subsystem having no neighbour $i-1$, the $1^{\text {st }}$ and $2^{n d}$ elements are equal to $2 \rho . C_{0}^{i}$ is the matrix containing the coefficients of the linear terms in $\mathcal{L}_{\rho}^{S}$ pertaining to $x_{i}$. The constraint reflects the lower bus voltage limits and is expressed in standard quadratic form where $A_{1}^{i}=2 \operatorname{diag}(1,1,0, \ldots, 0)$ so that $\frac{1}{2} x_{i}^{T} A_{1}^{i} x_{i}=V_{i}^{\prime} V_{i}$ and $D_{1}^{i}$ represents the lower limit of the normalized bus voltage magnitude squared.

The objective function of $\mathcal{P}_{U 1}$ is convex if the Hessian is positive semi-definite (i.e. $A_{0}^{i}+B_{0}^{i} \succeq 0$ ). As $A_{0}^{i}$ is the constant coefficient of the cost function $f_{i}($.$) , it is not guaranteed to be$ positive semi-definite (from Sec. II-C). Matrix $B_{0}^{i}$ contains $\rho$ terms in the diagonal elements which can be set to any nonnegative value. Thus, the value of $\rho$ is selected so that the Hessian $A_{0}^{i}+B_{0}^{i}$ is guaranteed to be positive semi-definite.

Theorem 1. When $\rho$ satisfies the following inequality, the objective function of $\mathcal{P}_{U 1}$ is convex:

$$
\rho_{U 1} \geq \max _{i \in n}\left\{\sqrt{\frac{1}{2}\left(\frac{R_{f i} K_{i}}{D_{i}}\right)^{2}+\frac{1}{4}\left(\frac{w L_{f i} K_{i}}{D_{i}}\right)^{2}}-\frac{1}{2}\left(\frac{R_{f i} K_{i}}{D_{i}}\right)\right\}
$$

Proof. This inequality has been derived from the premise that every eigenvalue of a positive semi-definite matrix is nonnegative [35]. Thus, $e$ is an eigenvalue of the matrix $A_{0}^{i}+B_{0}^{i}$ if $\operatorname{det}\left(A_{0}^{i}+B_{0}^{i}-\mathbf{I} e\right)=0$ where $\mathbf{I}$ is an identity matrix. The resulting roots of the equation are selected so that $e \geq 0$ from which the condition on $\rho$ listed in the above is derived. A detailed proof is listed in Appendix A of this paper. 
Although the objective is convex when $\rho$ satisfies the condition in Theorem 1, the non-convexity imposed by the bus voltage magnitude constraint must be overcome. We construct the dual function $g(\lambda)$ for $\mathcal{P}_{U 1}$ where $\lambda$ is the Lagrangian multiplier associated with the lower limit constraint:

$$
g(\lambda): \underset{x}{\operatorname{minimize}} \frac{1}{2} x^{T}\left(A_{0}+B_{0}\right) x+C_{0} x+\lambda\left(\underline{D_{1}}-\frac{1}{2} x^{T} A_{1} x\right)
$$

Closed-form expression can be obtained for $g(\lambda)$ by evoking the first-order optimality condition (i.e. $\frac{\partial \mathcal{L}_{U 1}}{\partial x}=0$ ) where $\mathcal{L}_{U 1}$ is the Lagrangian function associated with $\mathcal{P}_{U 1} . x$ can expressed in terms of $\lambda$ via the relation $\frac{\partial \mathcal{L}_{U 1}}{\partial x}=0$ :

$$
\begin{aligned}
& \frac{\partial \mathcal{L}_{U 1}}{\partial x}=\left(A_{0}+B_{0}\right) x+C_{0}-\lambda\left(A_{1} x\right)=0 ; \\
& x=-\left(A_{0}+B_{0}-\lambda A_{1}\right)^{-1} C_{0}
\end{aligned}
$$

where $x$ is in terms of $\lambda$. Substituting $x$ into $g(\lambda)$ results in the dual problem $\mathcal{D}_{U 1}$ :

$$
\begin{aligned}
\mathcal{D}_{U 1}: & \max _{\lambda_{U 1}}\left(D_{0}+\lambda \underline{D_{1}}\right)-C_{0}^{T}\left(A_{0}+B_{0}-\lambda A_{1}\right)^{-1} C_{0} \\
\text { s.t. } & \lambda \geq 0 \\
& A_{0}+B_{0}-\lambda A_{1} \succeq 0
\end{aligned}
$$

where constraint $\left(D 1^{\prime}\right)$ is necessary for any Lagrangian multiplier associated with an inequality constraint [35]. $\left(D 2^{\prime}\right)$ is a positive-semidefinite relation due to $\rho$ selected according to Theorem 1 . This constraint is necessary for avoiding the trivial optimal value of $-\infty$. The structure of the dual problem allows for the application of the Schur's complement which converts $\mathcal{D}_{U 1}$ to a semi-definite program $(\mathrm{SDP}) \mathcal{S}_{U 1}$ [36]:

$$
\begin{aligned}
\mathcal{S}_{U 1}: \underset{\lambda, \gamma}{\operatorname{maximize}} \quad \gamma & \\
\text { s.t. } & \lambda \geq 0 \\
& {\left[\begin{array}{cc}
A_{0}+B_{0}-\lambda A_{1} & C_{0} \\
C_{0}^{T} & D_{0}+\lambda D_{1}-\gamma
\end{array}\right] \succeq 0 }
\end{aligned}
$$

This concave dual problem can be solved to obtain the optimal value $\lambda^{*}$. This is then substituted back into the expression obtained for $x^{\prime}$ in terms of $\lambda$. The following theorem shows that this solution results in zero duality gap (i.e. solving $\mathcal{P}_{U 1}$ is equivalent to solving $\mathcal{S}_{U 1}$ ).

Theorem 2. Strong duality holds between $\mathcal{P}_{U 1}$ and $g$ where the dual optimal value $g^{*}$ is equal to the primal optimal value $\mathcal{P}_{U 1}^{*}$ when the primal problem $\mathcal{P}_{U 1}$ is strictly feasible.

Proof. For quadratically constrained quadratic program (QCQP) with a single constraint, the S-procedure which is commonly applied in control theory [37] states that if there exists a $\lambda \geq 0$ such that:

$$
\left[\begin{array}{cc}
A_{0}+B_{0} & C_{0} \\
C_{0}^{T} & D_{0}-\gamma
\end{array}\right]+\lambda\left[\begin{array}{cc}
-A_{1} & 0 \\
0 & D_{1}
\end{array}\right] \succeq 0
$$

then, the optimal values of the primal problem and the dual problem are equal regardless of whether the quadratic constraint is convex or not. This condition essentially imposes feasibility of the dual problem $\mathcal{S}_{U 1}$ which holds due to $\rho$ which is selected in accordance to Theorem 1.

\section{Summary of Proposed Algorithm}

Thus, since now all updates $(U 1)$ and $(U 2)$ are conducted via convex minimization operations, the proposed decentralized algorithm listed in Alg. 1 is guaranteed to converge via iterative refinement of local solutions by individual bus agents. The convergence rate is demonstrated to be linear by reference [17] with respect to the number of bus agents $n$ in the system (i.e. $\mathcal{O}(n)$ ). When an impending change in the demand and/or supply is about take place, the corresponding bus agent will trigger the distributed algorithm proposed in this paper by initiating the information exchange process. This will result in the computation of cost-effective setpoints of voltage source convertors that are able to accommodate the impending changes in the system in stead-state. Existing droop control methods can be implemented in conjunction with the proposed steady-state algorithm to address transience. As future work, we intend to explore combining optimal transient control with the proposed steady-state algorithm for all-encompassing microgrid coordination. This is illustrated via comprehensive simulation studies presented next.

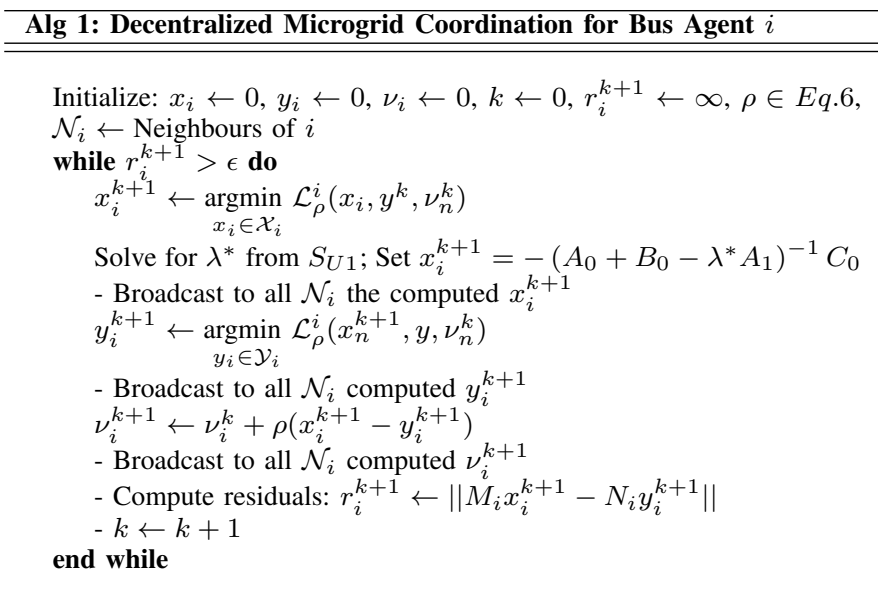

\section{RESULTS}

In this section, we validate the proposed decentralized algorithm via simulations involving realistic microgrid parameters obtained from reference [1]. Fig. 3 presents the line diagram for the 30-bus microgrid system utilized in the simulation studies. All simulations are implemented in MATLAB installed in a personal computer consisting of an i5 processor with 2.3 $\mathrm{GHz}$ speed.

\section{A. Steady-state Characteristics}

First, we establish that the steady-state characteristics of the linear dynamical microgrid system defined by the state-space equations in Eq. 1 correspond to the optimal values computed by the proposed algorithm in both the $d q 0$ and $a b c$ frames of references which are implemented in MATLAB and PSACD respectively. For this, a small microgrid containing three subsystems is implemented in PSCAD where the voltages $V_{t i}$ at each VSC are set to values computed by the proposed algorithm implemented in MATLAB. Fig. 4a contains the profile of bus voltage $V_{1}$ generated by the simulation over 0.3 seconds for sub-system 1 . We have added a data-tip for the time value 


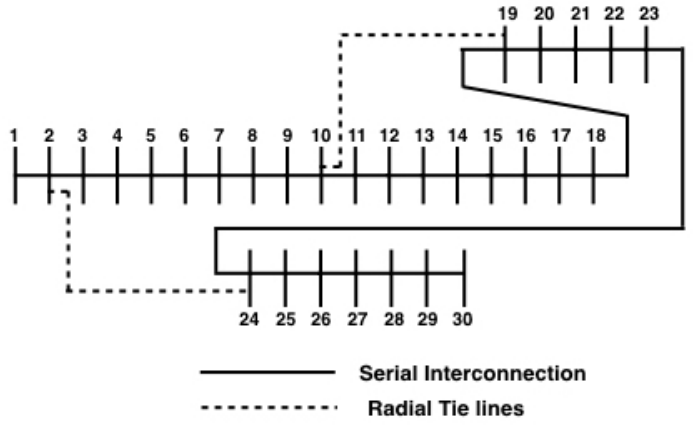

Fig. 3: 30-bus line diagram

of 0.2264 seconds in both the $a b c$ and $d q 0$ frame of references in Fig. 4a. It is clear that the magnitude of the point in the $d q 0$ frame which is $\sqrt{0.7033^{2}+0.7015^{2}}=0.9933$ is very close to the amplitude 0.9938 at that point in the $a b c$ frame which is the magnitude of the phasors. This minor discrepancy can be attributed to rounding errors and/differences in modelling dynamical systems in both platforms (i.e. MATLAB and PSCAD). Steady-state values generated in both frames of reference are identical and correspond to the optimal values computed by the proposed algorithm as expected. From Fig. $4 \mathrm{a}$, it is evident that the $d q 0$-frame eliminates the sinusoidal behaviour of the system in steady-state which is otherwise present in the $a b c$-frame.

\section{B. Convergence Characteristics}

Next, we assess the impact of the number of sub-systems present in the microgrid on the convergence characteristics of the proposed algorithm. For this, we have simulated four microgrids containing three, ten, twenty and thirty sub-systems respectively. The MATLAB simulations keep track of the total number of iterations necessary for convergence (i.e. when the residual is smaller than $\epsilon$ ). The convergence time is calculated by multiplying the worst case communication/computational delays associated with iteration. In each iteration $k$, three sets of information exchanges are necessary for the $x_{k}, y_{k}$ and $\nu_{k}$ updates. Each update entails the exchange of information with neighbouring buses which are typically one hop away. The worst case communication delay ranges from $8 \mathrm{~ms}$ to $30 \mathrm{~ms}$ [21] in one-hop communication in Zigbee Networks that are typically utilized in smart grid systems [20]. Computational complexity for $x$ and $y$ updates is associated with solving a quadratic problem or semi-definite program and this is typically $\mathcal{O}\left(b^{3}\right)$ as interior point methods are commonly utilized for solving these [35]. The $\nu$ update involves a simple linear combination. $b$ is the number of variables in each subproblem. As $|b|$ ranges between 5 to 10 variables per bus agent, solving these problems will entail a fraction of a millisecond with standard computational power available in distributed intelligent devices [20]. As per Sec. II-A, we allocate $100 \mathrm{~ms}$ for each update which includes sufficient margins for worst case communication and computational delays while also accounting for unexpected delays. Thus, each iteration takes place over a period of $0.1 s * 3=0.3 s$. Fig. $4 \mathrm{~b}$ illustrates how the residual evolves over the iterations $k$ for these systems. Convergence is achieved when the residual falls below $\epsilon$. It is clear that for larger microgrid systems, the number of iterations necessary for convergence increases as expected. In Fig. 4b, it is evident that for the 30-bus microgrid system, convergence entailed 160 iterations. Three sets of information exchanges are conducted every 0.3 seconds as per our system model listed in Sec. II-A. This translates to 48 seconds for convergence which allows for near real-time coordination of a system as large as the 30-bus microgrid.

We next study the impact of various values of $\rho$ on the convergence characteristics of the proposed system in Fig. 6a. Here we fix the microgrid to be a five-bus system and investigate how $\rho$ affects convergence. As per Theorem 1, $\rho$ is subject to a strictly positive lower limit. There is no upper limit imposed on $\rho$. According to reference [38], convergence of the ADMM algorithm is guaranteed for any strictly positive $\rho$ and convex formulation. For the results generated in Fig. 6a, the value of $\rho$ is changed from 10 to $10^{4}$ in multiples of 10 . It can be observed that the iterations for convergence does not increase significantly for $\rho=10^{4}$ from $\rho=10$ (i.e. by 75 iterations). It is clear that there are initial oscillations before the residual drops to a steady-state value for $\rho=10^{4} . \rho$ is a coefficient in $U 1$ and $U 2$ and imposes significant penalty in $x$ and $y$ updates. For the dual variable update in $U 3, \rho$ serves as the step-size. For large $\rho$, this results in the overshooting of the iterative updates of the dual variable. This can be attributed to the oscillations in the initial iterations. Then, the heavy penalty imposed on the $x$ and $y$ variables allow for the settling of the variables to the equilibrium values.

For typical microgrid systems (such as the one investigated in this paper), $\rho \approx 1.7$ for a microgrid consisting of any number of sub-systems. This is a very low value and since the system attributes are in terms per unit values, $\rho$ will not deviate significantly from this value. Thus, this value multiplied by a constant of 100 can be pre-set for all bus agents. Each bus agent can locally compute $\rho$ when there is a local change and if this value is larger than the current value, the corresponding agent can broadcast this value to all other agents in the system which can then be locally updated.

\section{System Limits}

The proposed coordination algorithm is premised upon accurately accounting for voltage-current balance and bus voltage magnitude limits with no relaxations in place. In order to ascertain that the proposed algorithm maintains system limits around acceptable thresholds even under severe system stress, bus loadings ranging from 1.0 p.u. to 2.0 p.u. are examined next. We specifically focus on increasing RL loads as these are major constituents of demands in micogrids that can lead to destabilizing stress in the system. In Fig. 5a we show that the VSC setpoints actuated in accordance to the values computed by the proposed algorithm maintains bus voltages within acceptable thresholds of $0.05 \pm 1$ p.u. Specifically, all buses voltages have been verified to lie within the acceptable thresholds and the confidence intervals of the voltage magnitude values taken by buses for various bus 

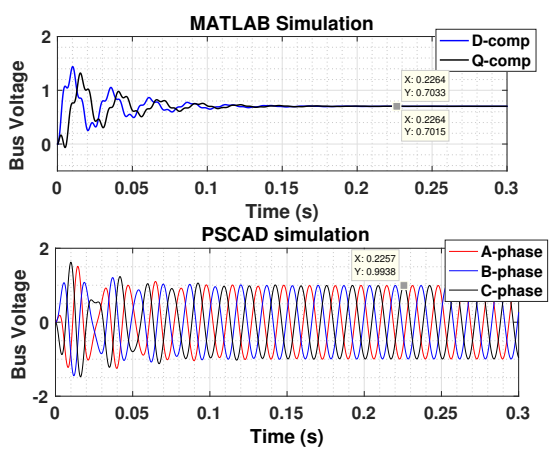

(a) PSCAD and MATLAB Comparison

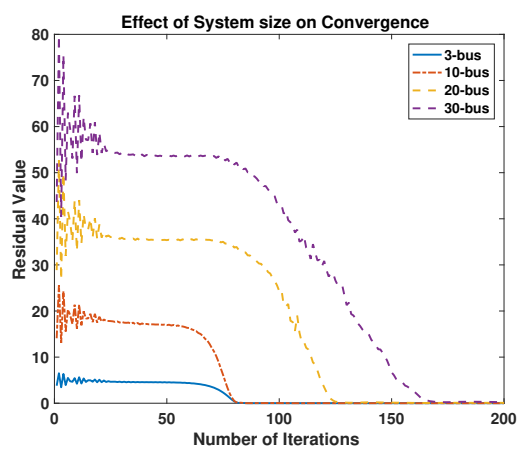

(b) Impact of System Size

Fig. 4: Convergence Study

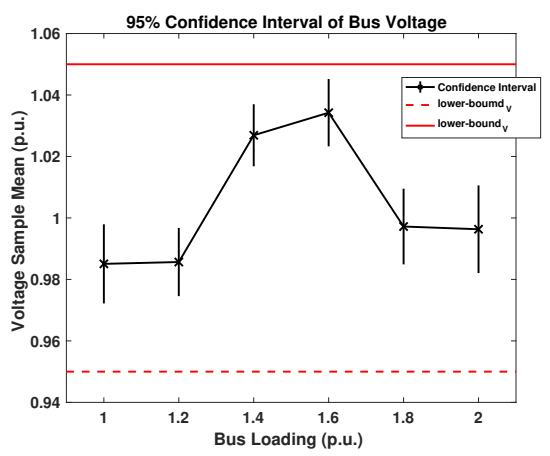

(a) Voltage regulation under changing load

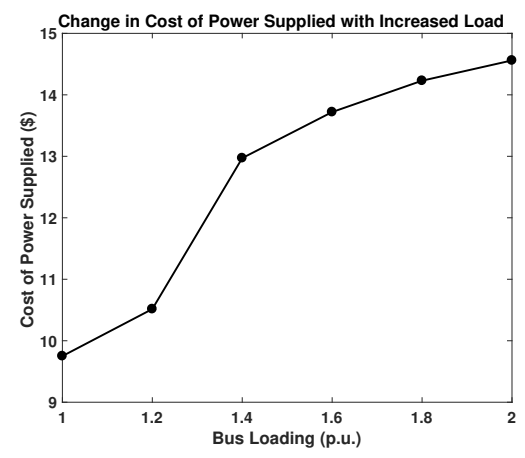

(b) Cost of Power with changing load

Fig. 5: Feasibility Study

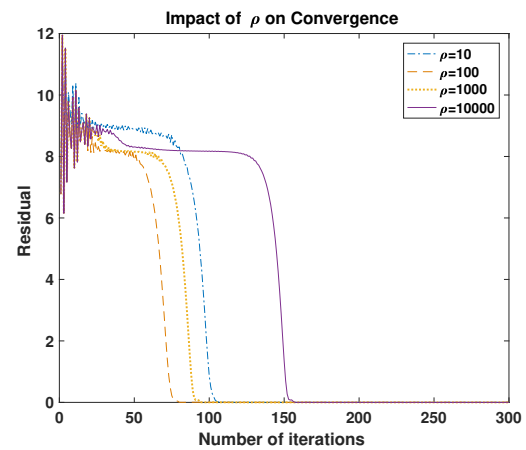

(a) Impact of $\rho$ on Convergence
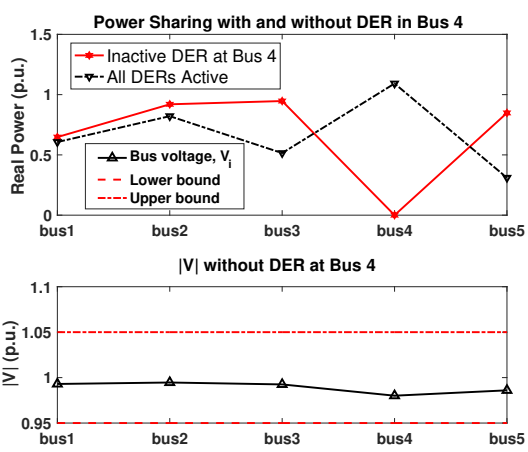

(b) Impact of Inactive DERs in buses

Fig. 6: System limits loadings show that the voltages are maintained around the nominal value of 1.0 p.u. We also investigate overall costs incurred in the system for increasing bus loading in Fig. 5b and as expected these costs increase approximately linearly with linearly rising demands. The costs $A_{i}$ assigned to DER generation are randomly generated positive values.

Next, we investigate the impact of inactive DERs in the microgrid system. In the original problem formulation $\mathcal{P}_{C}$, we have included constraint $(C 5)$ to accommodate instances where DERs are inactive in certain buses. The current injected by the inactive DER in the corresponding bus is set to 0 by the indicator variable in this constraint. Fig. $6 \mathrm{~b}$ illustrates the impact of the activation of this constraint in bus 4. As expected power sharing amongst DERs in other buses is adjusted accordingly when the DER in bus 4 is or is not active. Furthermore, the voltage profile in the system indicates a dip at bus 4 when the DER is not active as this bus is drawing power to supplement local loads and not injecting power back into the system.

\section{Comparative Studies}

Next, we present comparative studies of our proposal with recent state-of-the-art in the area of microgrid coordination. Specifically, we compare our work with the incremental costbased distributed droop strategy proposed in reference [15] which entails no information exchanges and utilizes only local measurements to improve local power dispatch by DERs. As this algorithm focuses mainly on real-time economic dispatch in microgrids, considerations that include bus voltage, reactive power and system limits are not included. When there is excessive bus loading or if the costs assigned to DERs are highly biased, it is possible for fundamental system limits to be violated. To illustrate this, we apply the algorithm proposed in [15] to a microgrid consisting of 5 sub-systems where the cost parameters $C_{i}$ are set to $(1000,1000,1000,0.01,1000)$ for each sub-system respectively. The DER in sub-system 4 has a significantly lower cost in comparison to other subsystems. Thus, intuitively, if there exists sufficient generation capacity, most power will be drawn from that source to supplement overall demands in the system if system limits are not considered. This is indeed the case as illustrated in Fig. 7a where significant real power is drawn from bus 4 . The bus voltages magnitudes are well above the acceptable upper limit of 1.05 p.u. as illustrated in Fig. 7b and this will result in equipment damages and cascading outages. Our algorithm, on the other hand, maintains bus voltages around the nominal value which is well within the safe operating limits. Thus, including underlying physical network characteristics allows for stable system operations. We also compare the performance of our proposal to the inbuilt non-convex quadratic program solver in MATLAB based on interior point method. Our proposal in this paper results in solutions that are associated with lower cost than that obtained by directly solving the nonconvex sub-problem. For instance, for a five-bus system, our proposal results in an overall cost of 0.80 whereas applying the non-convex solver for the same system incurs a cost of 1.18. This is expected as non-convex solvers do not guarantee optimality.

We also compare our proposal with recent literature in the area of microgrid coordination in Table I with respect 


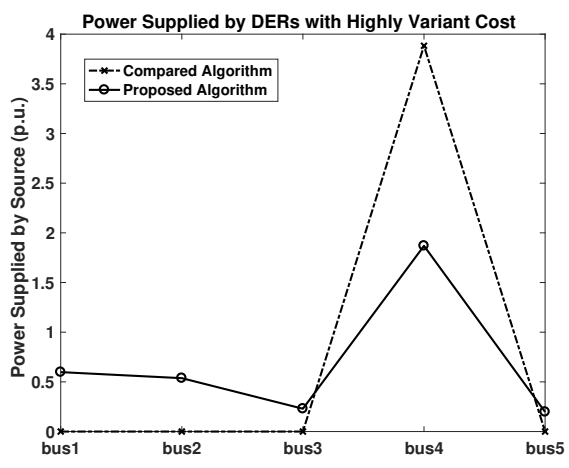

(a) Comparison study

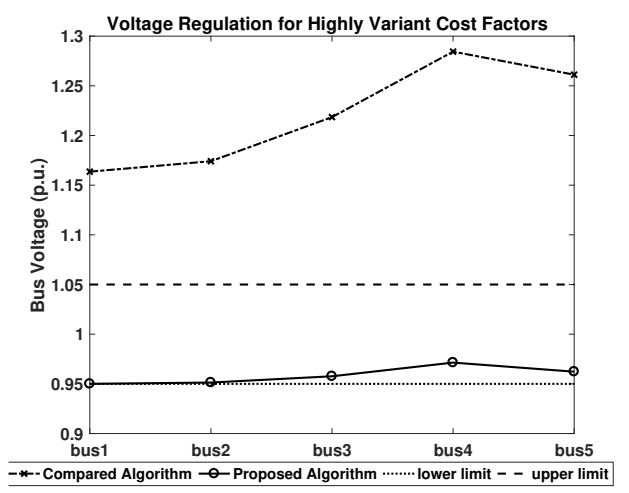

(b) Voltage regulation capability

Fig. 7: Comparison Studies

\begin{tabular}{|l||l|l|l|l|l|}
\hline & $\begin{array}{l}\text { Proposed } \\
\text { Algorithm }\end{array}$ & $\begin{array}{l}\text { Incremental-cost } \\
\text { droop algorithm [15] }\end{array}$ & $\begin{array}{l}\text { Distributed OPF algo- } \\
\text { rithm Type 1 [17] }\end{array}$ & $\begin{array}{l}\text { Distributed OPF Al- } \\
\text { gorithm Type 2 [19] }\end{array}$ & $\begin{array}{l}\text { Centralized } \\
\text { Algorithm in [10] }\end{array}$ \\
\hline \hline Convergence & Linear $\mathcal{O}(n)$ & - & Linear $\mathcal{O}(n)$ & Linear $\mathcal{O}(n)$ & $\mathcal{O}\left(n^{3}\right)$ \\
\hline $\begin{array}{l}\text { Communication } \\
\text { Overhead }\end{array}$ & $\mathcal{O}(m)$ & - & $\mathcal{O}(m)$ & $\mathcal{O}(m)$ & $\mathcal{O}(n)$ \\
\hline $\begin{array}{l}\text { Relaxations } \\
\text { of physical } \\
\text { microgrid } \\
\text { constraints }\end{array}$ & None & $\begin{array}{l}\text { Reactive power bal- } \\
\text { ance or voltage regu- } \\
\text { lation not considered }\end{array}$ & $\begin{array}{l}\text { SOCP inequality re- } \\
\text { laxation }\end{array}$ & $\begin{array}{l}\text { Optimal Solution, } X^{*} \\
\text { Rank relaxation }\end{array}$ & $\begin{array}{l}\text { Reactive power bal- } \\
\text { ance or voltage regu- } \\
\text { lation not considered }\end{array}$ \\
\hline $\begin{array}{l}\text { Feasibility in } \\
\text { Original OPF }\end{array}$ & Yes & Not guaranteed & Not guaranteed & Not guaranteed & Yes \\
\hline
\end{tabular}

TABLE I: Performance Comparison of Proposed Algorithm

to four attributes: 1) Convergence rate; 2) Communication overheads; 3) Relaxations; and 4) Guarantees of feasibility. We have examined proposals that entail no communication [15], decentralized computations based on peer-to-peer information exchanges [17], [19] and centralized solution [10]. We denote the total number of DERs in the system as $n$ and the number of neighbouring nodes as $m$. The convergence rate of decentralized solutions (ours and [17], [19]) is $\mathcal{O}(n)$ as these are convex formulations that utilize information exchanges designed using ADMM. For centralized methods [10], the computational complexity entailed is $\mathcal{O}\left(n^{3}\right)$. All solutions involving peerto-peer information exchanges incur a communication complexity of $\mathcal{O}(\mathrm{m})$. Next, we examine the relaxations applied to the formulation of the optimal power flow problem in the microgrid in these proposals. We apply no relaxations while references [17] and [19] apply second order cone (SOC) and rank relaxations respectively. If the solutions produced by these proposals do not satisfy the original OPF constraints, then these are infeasible. Proposals [15] and [10] completely neglect reactive power balance and voltage regulation and these are also subject to in-feasibilities. Thus, although our proposal entails communication with neighbouring nodes, the superior convergence characteristics render it ideal for near real-time microgrid coordination.

The afore-mentioned strategies are implemented in $\mathrm{AC}$ systems. DC microgrids are also becoming prevalent and reference [41] is an example of work in this area that investigates fair load sharing in a decentralized manner while maintaining actuation constraints such as voltage limits and so on. The fundamental differences between this work and our work are due to the fact that the underlying system is different (i.e. DC vs AC). The problem formulation in [41] is a convex quadratic program with linear constraints. In our work, although we apply the Park's transformation to construct linear current and voltage balance constraints, the voltage magnitude constraints are quadratic and the cost function is also quadratic. As the state variables are voltage and current, expressing power in terms of these variables result in non-convexities in the objective function. Furthermore, the lower limit on the bus voltage magnitude constraint is also non-convex. Thus, our work deals with these additional challenges by proposing a decentralized algorithm based on Schur's complement and the $\mathrm{S}$-procedure that is able to exactly overcome these.

Other approaches like references [42], [43] separate the microgrid coordination problem into multiple hierarchies. Optimal dispatch values are computed at the secondary level via methods that include consensus and primal-dual decomposition. Then, primary control techniques such as AC frequency, voltage and power droop control are utilized to offset fluctuations in the system. These droop techniques may result in sub-optimality before the next optimal dispatch setpoint is computed at the secondary level. Economic dispatch formulated at the secondary level does not account for voltage and system losses. Our work differs from these approaches as we exactly account for power losses and system limits (e.g. voltage magnitude limits, etc.) at a highly granular manner and thus closely accounting for fluctuations in near real-time. 


\section{CONCLUSIONS}

We have proposed a decentralized microgrid coordination algorithm for near real-time computations of optimal VSC set-points which can readily adopt to ongoing changes in the system. This algorithm accounts for the underlying electrical constructs of the microgrid which is imperative for maintaining feasible and stable system operations. We have leveraged on the linear steady-state voltage/current balance relations in the $d q 0$ frame to formulate the optimal microgrid coordination problem and strategically applied ADMM to decompose this problem into simpler sub-problems that allows for the application of an exact convex relaxation via the Schur's complement and S-procedure. We have highlighted through practical simulation and comparative studies the convergence, performance and feasibility characteristics of our proposal under various system settings. As future work, we intend to investigate how this algorithm can be used in tandem with effective transient control strategies for allowing efficient and stable control of microgrid systems. This initiative is a significant step in realizing an efficient, sustainable and responsive energy landscape.

\section{APPENDIX: ProOf OF TheOREM 1}

In this section, we present the proof of Theorem 1 that presents a lower limit on $\rho$ that guarantees the convexity of the objective of $\mathcal{P}_{U 1}$. The original objective of $\mathcal{P}_{U 1}$ is:

$$
f_{i}\left(x_{i}\right)=\frac{1}{2} x_{i}^{T}\left(A_{0}^{i}+B_{0}^{i}\right) x_{i}+C_{0}^{i} x_{i}
$$

The non-convexity in the above objective is due to the quadratic terms associated with $V_{i}$ and $V_{t i}$ (based on Eq. 2). We specifically consider these quadratic terms next:

$$
\begin{aligned}
& f_{i}\left(V_{i, d q}, V_{t i, d q}\right)=\left[\begin{array}{ll}
V_{i, d q} & V_{t i, d q}
\end{array}\right]^{T}\left(A_{S 0}^{i}+B_{S 0}^{i}\right)\left[\begin{array}{ll}
V_{i, d q} & V_{t i, d q}
\end{array}\right] \\
& \text { where } A_{S 0}^{i}=\frac{\frac{3}{2} K_{i}}{R_{f i}^{2}+\left(\omega L_{f i}\right)^{2}}\left[\begin{array}{cccc}
0 & 0 & -\frac{R_{f i}}{\omega 2_{f i}} & \frac{\omega L_{f i}}{2_{f i}} \\
0 & 0 & -\frac{R_{f i}}{2} & -\frac{R_{f i}}{2} \\
-\frac{\omega L_{f i}}{2} & -\frac{R_{f i}^{2}}{2} & R_{f i} & 0 \\
\frac{R_{f i}^{2}}{2} & 0 & R_{f i}
\end{array}\right]
\end{aligned}
$$$$
\text { and }\left(B_{S 0}^{i}\right)_{\text {worst case }}=2 \operatorname{diag}(\rho, \rho, \rho, \rho)
$$

We set the worst case $B_{S 0}^{i}$ to be $2 \operatorname{diag}(\rho, \rho, \rho, \rho)$ which occurs for the root bus in the physical network topology representing the microgrid. For all other buses, the first two diagonal elements in $B_{S 0}^{i}$ are $4 \rho$. Since, we are utilizing $\rho$ to maintain the convexity of the objective, we are seeking the lower limit that is applicable for all buses. For convexity of the objective to hold, the constant matrix associated with the $f_{i}\left(V_{i, d q}, V_{t i, d q}\right)$ term must be positive semi-definite [35] (i.e. $\left(A_{S 0}^{i}+B_{S 0}^{i}\right) \succeq 0$ ) which translates to:

$$
\begin{aligned}
& {\left[\begin{array}{cccc}
\rho & 0 & -\frac{a-\rho}{2} & \frac{b}{2} \\
0 & \rho & -\frac{b}{2} & -\frac{a-\rho}{2} \\
-\frac{a-\rho}{2} & -\frac{b}{2} & a & 0 \\
\frac{b}{2} & -\frac{a-\rho}{2} & 0 & a
\end{array}\right] \succeq 0 \text { where, }} \\
& a=\rho+R_{f i} \cdot \frac{\frac{3}{2} K_{i}}{R_{f i}^{2}+\left(\omega L_{f i}\right)^{2}}, b=\omega L_{f i} \cdot \frac{\frac{3}{2} K_{i}}{R_{f i}^{2}+\left(\omega L_{f i}\right)^{2}}
\end{aligned}
$$

Positive semi-definiteness of a square matrix can be assessed by examining the eigenvalues of the matrix. If these are non-negative, then positive semi-definiteness holds. Let the eigenvalue be represented as $e$ and this can be computed by solving the characteristic equation: $\operatorname{det}\left(A_{0}^{i}+B_{0}^{i}-\mathbf{I} e\right)=0$. With some algebraic manipulations, we obtain the following relation for the characteristic equation:

$$
\left[(\rho-e)(a-e)-\frac{(a-\rho)^{2}}{4}-\frac{b^{2}}{4}\right]^{2}=0
$$

It is clear that the above can be expanded to a fourth-degree polynomial in terms of $e$ and this characteristic equation has two pairs of repeated roots (i.e. there are 4 eigenvalues). Applying the quadratic formula, each pair of repeated eigenvalues can be computed as follows:

$$
e_{\text {repeated }}=\frac{(a+\rho) \pm \sqrt{(a+\rho)^{2}-4\left\{a \rho-\frac{(a-\rho)^{2}}{4}-\frac{b^{2}}{4}\right\}}}{2}
$$

For positive semi-definiteness to hold, the eigenvalues must be non-negative. To enforce this condition, it suffices to set this condition on the smallest eigenvalue as follows:

$$
\frac{(a+\rho)-\sqrt{(a+\rho)^{2}-4\left\{a \rho-\frac{(a-\rho)^{2}}{4}-\frac{b^{2}}{4}\right\}}}{2} \geq 0
$$

Since $\rho$ can be selected to enforce the above condition, we isolate $\rho$ in the above inequality to obtain:

$$
\begin{aligned}
& \rho \geq \sqrt{\frac{1}{2}\left(R_{f i} \cdot \frac{\frac{3}{2} K_{i}}{R_{f i}^{2}+\left(\omega L_{f i}\right)^{2}}\right)^{2}+\frac{1}{4}\left(\omega L_{f i} \cdot \frac{\frac{3}{2} K_{i}}{R_{f i}^{2}+\left(\omega L_{f i}\right)^{2}}\right)^{2}} \\
& -\frac{1}{2}\left(R_{f i} \cdot \frac{\frac{3}{2} K_{i}}{R_{f i}^{2}+\left(\omega L_{f i}\right)^{2}}\right)
\end{aligned}
$$

Setting $D_{i}$ to be $\frac{2}{3}\left(R_{f i}^{2}+\left(\omega L_{f i}\right)^{2}\right)$, we obtain the lower limit listed in Theorem 1 .

\section{REFERENCES}

[1] A.H. Etemadi, E.J. Davison, and R. Iravani, "A decentralized robust control strategy for multi-der microgrids-part I: Fundamental concepts," IEEE Transactions on Power Delivery, vol. 27, no. 4, pp. 1843-1853, Oct. 2012.

[2] J.M. Guerrero, J.C. Vasquez, J. Matas, L.G. de Vicuña, and M. Castilla, "Hierarchical control of droop-controlled AC and DC microgrids: A general approach toward standardization," IEEE Transactions on Industrial Electronics, vol. 58, no. 1, pp. 158-172, Jan. 2011.

[3] Q. Shafiee, J. M. Guerrero, and J. C. Vasquez, "Distributed secondary control for islanded microgrids- A novel approach," IEEE Transactions on Power Electronics, vol. 29, no. 2, pp. 1018-1031, Feb. 2014.

[4] A. Mehrizi-Sani and R. Iravani, "Constrained potential function based control of microgrids for improved dynamic performance," IEEE Transactions on Smart Grid, vol. 3, no. 4, pp. 1885-1892, Dec. 2012.

[5] V. Nasirian, Q. Shafiee, J. Guerrero, F. L. Lewis and A. Davoudi, "Droop-free distributed control for AC microgrids," IEEE Transactions on Power Electronics, vol. 31, no. 2, pp. 1600-1617, Feb. 2016.

[6] T.L. Vandoorn, J.D.M. De Kooning, B. Meersman, and L. Vandevelde, "Review of primary control strategies for islanded microgrids with power electronic interfaces," Renewable and Sustainable Energy Reviews, vol. 19, pp. 613-628, Mar. 2013.

[7] D. E. Olivares, A. Mehrizi-Sani, A.H. Etemadi, C. Cañizares, R. Iravani, M. Kazerani, A.H. Hajimiragha, O. Gomis-Bellmunt, M. Saeedifard, R. Palma-Behnke et al., "Trends in microgrid control," IEEE Transactions on Smart Grid, vol. 5, no. 4, pp. 1905-1919, Jul. 2014.

[8] S. S. Soman, H. Zareipour, O. Malik and P. Mandal, "A review of wind power and wind speed forecasting methods with different time horizons," in Proc. of IEEE North American Power Symposium, Arlington, TX, USA, Sep. 2010. 
[9] M. Marzband, F. Azarinejadian, M. Savaghebi, and J.M. Guerrero, "An optimal energy management system for islanded microgrids based on multiperiod artificial bee colony combined with markov chain," IEEE Systems Journal, vol. 11, no. 3, pp. 1712-1722, Sep. 2017.

[10] M. Ross, C. Abbey, F. Bouffard, and G. Jos, "Multiobjective optimization dispatch for microgrids with a high penetration of renewable generation," IEEE Transaction on Sustainable Energy, vol. 6, no. 4, pp. 1306-1314, Oct. 2015.

[11] J.A.P Lopes, C.L. Moreira and A.G. Madureira, "Defining control strategies for microgrids islanded operation," IEEE Transactions on power systems , vol. 21, no. 2, pp. 916-924, May 2006

[12] S.H. Low, "Convex relaxations of optimal power flow part I: Formulations and equivalence," IEEE Transactions on Control Network Systems, vol. 1, no. 1, pp. 15-27, Mar. 2014

[13] S. Huang, Q. Wu, J. Wang, and H. Zhao, "A sufficient condition on convex relaxation of AC optimal power flow in distribution networks," IEEE Transactions on Power Systems, vol. 32, no. 2, pp. 1359-1368, Mar. 2017.

[14] Yinliang Xu, Zhicheng Li, "Distributed optimal resource management based on the consensus algorithm in a microgrid," IEEE Transactions on Industrial Electronics, vol. 62, no. 4, pp. 2584-2592, Apr. 2015.

[15] F. Chen, M. Chen, Q. Li, K. Meng, and Y. Zheng, J.M. Guerrero, and D. Abbott, "Cost-based droop schemes for economic dispatch in islanded microgrids," IEEE Transactions on Smart Grid, vol. 8, no. 1, pp. 63-74, Jan. 2017.

[16] B. Zhang , A.Y. Lam, A.D. Garcia and D.Tse, "An optimal distributed method for voltage regulation in power distribution systems." IEEE Transactions on Power Systems, vol. 30, no. 4, pp. 1714-1726, Jul. 2015.

[17] Q. Peng and S.H. Low, "Distributed algorithm for optimal power flow on a radial network," in Proc. of the 53rd IEEE Annual Conference on Decision and Control, CA, USA, Dec. 2014, pp. 167-172.

[18] T. Erseghe, "Distributed optimal power flow using ADMM," IEEE Transactions on Power System, vol. 29, no. 5, pp. 2370-2380, Sep. 2014.

[19] E. Dallanese, H. Zhu, and G.B. Giannakis, "Distributed optimal power flow for smart microgrids," IEEE Transactions on Smart Grid, vol. 4, no. 3, pp. 1464-1475, Sep. 2013.

[20] H. Farhangi, "The path of the smart grid," IEEE Power Energy Magazine, vol. 8, no. 1, pp. 18-28, Jan./Feb. 2010

[21] B. Krupanek and R. Bogacz, Investigations of transmission delays in ZigBee networks, Politechnika Slaska, Instytut Metrologii, Elektroniki i Automatyki, vol. 1, no. 2, 2014.

[22] N.W.A. Lidula and A.D. Rajapakse, "Microgrids research: A review of experimental microgrids and test systems," Renewable and Sustainable Energy Reviews, vol. 15, no. 1, pp. 186-202, Jan. 2011.

[23] IEEE Guide for Design, Operation, and Integration of Distributed Resource Island Systems with Electric Power Systems, IEEE Standard 1547.4, Jul. 2011.

[24] GC Liao, JI Tsai "Use of a new method to solve the economic dispatch problem of smart microgrid including distributed generation," in Proc. of Asia-Pacific Power and Energy Engineering Conference, Shanghai, China, Mar. 2012, pp. 1-4.

[25] R. Majumder; A. Ghosh; G. Ledwich; F. Zare, "Control of parallel converters for load sharing with seamless transfer between grid connected and islanded modes," in Proc. of IEEE Power and Energy Society General Meeting, Pittsburgh, PA, USA, Jul. 2008, pp. 1-7.

[26] C.L. Masters, "Voltage rise: the big issue when connecting embedded generation to long $11 \mathrm{kV}$ overhead lines," IET Power Engineering journal, vol. 16, no. 1, pp. 5-12, Feb. 2002.

[27] P. Krause; O. Wasynczuk, SD Sudhoff, S. Pekarek, Analysis of Electric Machinery and Drive System New York, USA: IEEE Press, 2002.

[28] IEEE Standard for Synchrophasors for Power Systems, IEEE Standard C37.118-2005, 2006, (Revision of IEEE Standard 1344-1995).

[29] A.G. Phadke, J. Thorp, Synchronized Phasor Measurements and Their Applications, 1st ed. New York, USA: Springer, 2008.

[30] J. Vig, "Quartz crystal resonators and oscillators for frequency control and timing applications: A tutorial," U.S. Army CommunicationsElectronics Command, Fort Monmouth, NJ, USA, Tech. Rep. SLCETTR-88-1 (Rev. 8.4.2), 2000.

[31] H. Lotfi and A. Khodaei, "AC versus DC microgrid planning," IEEE Transactions on Smart Grid, vol. 8, no. 1, pp. 296-304, Jan. 2017.

[32] F. Luo, Z. Y. Dong, G. Liang, J. Murata, Z. Xu, "A distributed electricity trading system in active distribution networks based on multi-agent coalition and blockchain", IEEE Transactions on Power Systems, 2019. (Early access article)

[33] R. Reginatto and R. A. Ramos, "On electrical power evaluation in dq coordinates under sinusoidal unbalanced conditions," IET Generation, Transmission \& Distribution, vol. 8, no. 5, pp. 976-982, May 2014
[34] G.P. Adam, O.A. Lara, G.M. Burt, D. Telford, B.W. Williams, and J.R. McDonald, "Modular multilevel inverter: Pulse width modulation and capacitor balancing technique," IET Power Electronics, vol. 3, no. 5, pp. 702-715, Sept. 2010.

[35] S. Boyd and L. Vandenberghe. Convex Optimization . Cambridge, UK: Cambridge university press, 2004.

[36] J. A. Taylor. Convex Optimization of Power Systems. Cambridge, UK: Cambridge University Press, 2015.

[37] U. T. Jonsson. (2001). "A Lecture on the S-procedure [Online]. Available: https://people.kth.se/ uj/5B5746/Lecture.ps."

[38] S. Boyd, N. Parikh, E. Chu, B. Peleato, and J. Eckstein, "Distributed optimization and statistical learning via the alternating direction method of multipliers," Foundations and Trends in Machine Learning, vol. 3, no. 1, pp. 1-122, July 2011.

[39] J. Park; S. Boyd, "General heuristics for nonconvex quadratically constrained quadratic programming," arXiv:1703.07870, 2017.

[40] A. Ben-Tal and M. Teboulle, "Hidden convexity in some nonconvex quadratically constrained quadratic programming," Mathematical Programming, vol. 72, no. 1, pp. 51-63, Jan. 1996

[41] J. Zhao, F. Dorfler, "Distributed control and optimization in DC microgrids," Automatica, vol. 61, pp. 18-26, Nov. 2015.

[42] P. Lin, C. Jin, J. Xiao, X. Li, D. Shi, Y. Tang and P. Wang, "A distributed control architecture for global system economic operation in autonomous hybrid AC/DC microgrids," IEEE Transactions on Smart Grid,vol. 10, no. 3, pp. 2603-2617, May 2019.

[43] P. Vergara, J. Rey, H. Shaker, J. Guerrero, B. J. Jorgensen and L. Silva, "Distributed strategy for optimal dispatch of unbalanced three-phase islanded microgrids," IEEE Transactions on Smart Grid, vol. 10, no. 3, pp. 3210-3225, May 2019.

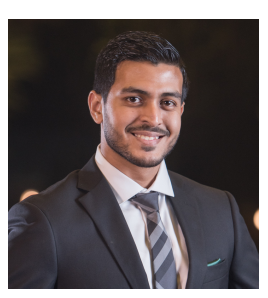

Mithun Mallick received his B.Sc. degree from the Electrical \& Electronic Engineering department of Bangladesh University of Engineering \& Technology, Dhaka, Bangladesh in 2017. He is currently pursuing the M.E. Sc. degree from Department of Electrical \& Computer Engineering at Western University, Canada. He is also a student member of the IEEE Power \& Energy Society. His research interests lie in the area of optimization, protection and control of microgrids, smart grids, renewable energy systems and large-scale power systems. He is also keen in exploring the applications of various numerical methods and game theory in power systems.

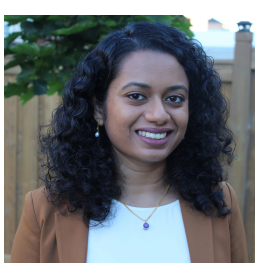

Pirathayini Srikantha is currently an Assistant Professor in the Department of Electrical and Computer Engineering at Western University. She received her B.A.Sc. degree in Systems Design Engineering from the University of Waterloo in 2009 and her M.A.Sc. degree in Electrical and Computer Engineering from the same institute in 2013. She obtained her Ph.D. degree from The Edward S. Rogers Sr. Department of Electrical and Computer Engineering at the University of Toronto in 2017. She is a certified Professional Engineer (P.Eng.) in Ontario. Her main research interests are in the areas of large-scale optimization and distributed control for enabling adaptive, sustainable and resilient power grid operations. Her work has been published in premier smart grid journal and conference venues. Her research efforts have received recognitions that include the best paper award (IEEE Smart Grid Communications) and runner-up best poster award (ACM Women in Computing). She is also actively involved in professional and social activities. She has served as the Workshop Chair, Session Chair and Technical Program Committee member in IEEE conferences. She is a reviewer in numerous IEEE transactions journals. 